\title{
TECNOB Study: Ad Interim Results of a Randomized Controlled Trial of a Multidisciplinary Telecare Intervention for Obese Patients with Type-2 Diabetes
}

\author{
Gianluca Castelnuovo ${ }^{1,3, *}$, Gian Mauro Manzoni ${ }^{1,2}$, Paola Cuzziol ${ }^{1,2}$, Gian Luca Cesa ${ }^{1}$, Stefania \\ Corti $^{1}$, Cristina Tuzzi ${ }^{4}$, Valentina Villa ${ }^{1}$, Antonio Liuzzi ${ }^{5}$, Maria Letizia Petroni ${ }^{4}$ and Enrico \\ Molinari $^{1,3}$ \\ ${ }^{I}$ Istituto Auxologico Italiano IRCCS, Psychology Research Laboratory, Ospedale San Giuseppe, Verbania, Italy \\ ${ }^{2}$ Department of Psychology, University of Bergamo, Italy \\ ${ }^{3}$ Department of Psychology, Catholic University of Milan, Italy \\ ${ }^{4}$ Istituto Auxologico Italiano IRCCS, Laboratory of Nutrition Research, Ospedale San Giuseppe, Verbania, Italy \\ ${ }_{5}^{5}$ Istituto Auxologico Italiano IRCCS, Diabetes Research Laboratory, Ospedale San Giuseppe, Verbania, Italy
}

\begin{abstract}
Background: Obesity increases the risk of many health complications such as hypertension, coronary heart disease and type 2 diabetes, needs long-lasting treatment for effective results and involves high public and private care-costs. Therefore, it is imperative that enduring and low-cost clinical programs for obesity and related co-morbidities are developed and evaluated. Information and communication technologies (ICT) can help clinicians to deliver treatment in a costeffective and time-saving manner to a large number of obese individuals with co-morbidities.
\end{abstract}

Objective: To examine ad interim effectiveness of a 12-month multidisciplinary telecare intervention for weight loss provided to obese patients with type 2 diabetes.

Design, Setting, and Participants: A single-center randomized controlled trial (TECNOB study) started in December 2008. At present, 72 obese patients with type 2 diabetes have been recruited and randomly allocated to the TECNOB program $(n=37)$ or to a control condition $(n=39)$. However, only 34 participants have completed at least the 3-month followup and have been included in this ad interim analysis. 21 out of them have reached also the 6-month follow-up and 13 have achieved the end of the program. Study is still on-going.

Intervention: All participants attended 1-month inpatient intensive program that involved individualized medical care, diet therapy, physical training and brief psychological counseling. At discharge, participants allocated to the TECNOB program were instructed to use a weight-loss web-site, a web-based videoconference tool, a dietary software installed into their cellular phones and an electronic armband measuring daily steps and energy expenditure.

Main Outcome Measures: Weight and disordered eating-related behaviors and cognitions (EDI-2) at entry to hospital, at discharge from hospital, at 3,6 and 12 months.

Results: Ad interim analysis of data from 34 participants showed no statistically significant difference between groups in weight change at any time-point. However, within-group analysis revealed significant reductions of initial weight at discharge from hospital, at 3 months, at 6 months but not at 12 months. Control group had higher scores in Interpersonal distrust at 12 months.

Conclusion: This ad interim findings revealed that the effect of the inpatient treatment was high and probably overwhelmed the effect of the TECNOB intervention. Much statistical power and long-term follow-up may enhance the probability to detect the TECNOB effect over and above the great one exerted by the inpatient program.

Keywords: Obesity, type-2 diabetes, weight loss, weight loss maintenance, telemedicine, telecare, RCT.

\section{BACKGROUND}

Obesity is a condition with such an increasing prevalence that it can be defined as a global epidemic. In 2005, about

*Address correspondence to this author at the Psychology Research Laboratory, San Giuseppe Hospital, Istituto Auxologico Italiano, Via Cadorna, 90, 28824 Piancavallo (VB), Italy; Tel: 0039323 514338;

Fax: 0039323 514338; E-mail: gianluca.castelnuovo@auxologico.it
1.6 billion adults (above 15 years of age) were estimated to be overweight, whereas about 400 million people were obese. WHO further projects that by 2015 , approximately 2.3 billion adults will be overweight and more than 700 million will be obese [1]. Obesity increases the risk of many health complications such as cardiovascular diseases, some types of cancer, osteoarthritis, hypertension, dyslipidemia, and hypercholesterolemia, and is associated with early death $[2,3]$. Obesity is a strong risk factor for the development of 
type II diabetes [4, 5]. Indeed, as BMI (Body Mass Index) increases, the risk of developing type 2 diabetes increases in a "dose-dependent" manner [6,7]. The prevalence of type 2 diabetes is 3-7 times higher in obese than in normal-weight adults, and those with a BMI $>35$ are 20 times more likely to develop type 2 diabetes than those with a BMI between 18.5 and $24.9[8,9]$.

Obesity-related medical complications weigh heavily on public health care costs and developing effective interventions for substantially reduce weight, maintain weight loss and prevent or manage associated diseases like type 2 diabetes in cost-effective manner is a priority.

Stand-alone and combined treatment options (dietetic, nutritional, physical, behavioral, cognitive-behavioral, pharmacological, surgical) are available, but clinical practice and research have shown significant difficulties with regard to availability, costs, treatment adherence and long-term efficacy [10]. These procedures imply high costs both for the obese individuals and the public health system, overall within an enduring care setting. Indeed, the main challenge in the treatment of obesity is to maintain weight loss in the long term [11]. Most overweight and obese individuals regain about one third of the weight lost with treatment within 1 year, sometimes even before the end of the intervention, and they are typically back to baseline in 3 to 5 years [1214]. Similarly, few patients with diabetes go on taking their prescribed medication entirely as intended $[15,16]$.

Continuous and cost-effective approaches that can reach a large number of obese individuals are thus needed. A new promising method for granting continuity of care to wide populations of patients at low costs is telemedicine and its more specific branches called "e-therapy", "telecare" and "ehealth": information and communication technologies (ICT) used in order to exchange information useful for the diagnosis, treatment, rehabilitation and prevention of diseases [17, 18]. Telecare can be carried out with tools such as web-sites, e-mail, chat lines, videoconference, telephone and mobile phones [19]. As already indicated in several studies [20-24] and in various reviews $[10,25,26]$, behavioral treatments delivered through the internet (web-site and e-mail) may be valid alternatives to reduce expensive and time-consuming clinical visits.

However, much more work remains to be carried out in order to confirm these findings. Furthermore, very few studies have investigated the effects of an ICT-based program on obese patients with established type 2 diabetes and to our knowledge no study has tested a comprehensive long-term stepped down intervention starting with 1-month hospitalization specifically addressed to weight loss. Thus, we planned to test the effectiveness of a 12-month multidisciplinary telecare intervention for weight loss in comparison with a control condition. Study is still ongoing and ad interim results were reported in this paper.

\section{MATERIALS AND METHODOLOGY}

\section{Study Sample}

At present, 72 adult obese patients with a mean age of $53,3 \pm 8,4$ years, a mean weight of $120,25 \pm 20,8 \mathrm{~kg}$ and type-2 diabetes mellitus have been recruited by research assistants at admission to a 1-month inpatient program for weight loss and medical care. Potential participants have been identified from notice given by physicians after they established a type- 2 diabetes mellitus diagnosis. Further inclusion criteria were age between 18 and 65 years old, a body mass index $(\mathrm{BMI}) \geq 30$, basic knowledge of informatics and home access to a computer with Internet service. Moreover, patients had to be free of severe psychiatric disturbances diagnosed by DSM-fourth revised criteria and concurrent medical conditions not related to obesity. Eligible candidates were scheduled for a 1-hour interview with a clinical psychologist during which their psychosocial status was assessed, weight and dieting histories were reviewed, study details were described and informed consent was obtained.

At present, only 34 patients have completed the 3-month follow-up and have been considered for this first ad interim analysis. 21 patients out of them have come through the 6month follow-up and 12 have achieved the end of program (12-month follow-up). 23 patients out of 72 have been lost at 3-month follow-up because of many reasons such as surgery, house transfer, repeated loss of mailed questionnaires, critical life-events, motivation loss, etc.

\section{Study Design}

A detailed description of the study protocol is available elsewhere [27]. Briefly, eligible patients who accepted participation were randomly assigned according to simple randomization procedures (computerized random numbers) to the TECNOB program or to a control condition. Participants were seen at baseline and at discharge from hospital for objective measurement of weight and questionnaires administration. Follow-up assessments at 3, 6 and 12 months were self-reported. All participants attended 1-month inpatient program for weight loss and medical care. Before discharge, participants allocated to the TECNOB group were instructed to use a weight-loss web-site, a dietary software installed into their cellular phones, a web-based videoconference tool and an electronic armband measuring daily steps and energy expenditure. To protect confidentiality, the web-site was password protected and participants were given a login identification code. Patients assigned to control group did not receive any instructions and were discharged as all the other inpatients not included in the study.

The research protocol was approved by the institutional review board at Istituto Auxologico Italiano, Italy.

\section{Program Description}

The TECNOB (TEChnology for OBesity) program is a comprehensive two-phase stepped down intervention enhanced by telecare for the long-term treatment of obese people with type 2 diabetes seeking treatment for weight loss [28]. The core aspects of TECNOB are the inpatient intensive treatment and the continuity of care at home using new information and communication technologies (ICT) such as internet and mobile phones.

The TECNOB clinical program has a total duration of 13 months and consists of two stepped down phases: inpatient (1 month) and outpatient (the following 12 months). During the inpatient phase, participants attend an intensive four week and medically-managed program for weight reduction and rehabilitation. All patients are placed on a hypocaloric 
nutritionally balanced diet tailored to the individual after consultation with a dietitian (energy intake around $80 \%$ of the basal energy expenditure estimated according to the Harris-Benedict equation and a macronutrient composition of $16 \%$ proteins, $25 \%$ fat and $59 \%$ carbohydrates). Furthermore, they receive nutritional counseling provided by a dietitian, brief psychological counseling provided by a clinical psychologist and have physical activity training provided by a physiotherapist.

Nutritional rehabilitation program aims to improve and promote change in eating habits and consists of both individual sessions (dietary assessment, evaluation of nutrient intake and adequacy, nutritional status, anthropometric, eating patterns, history of overweight, readiness to adopt change) and group sessions (45 minutes each twice a week) including: information on obesity and related health risks, setting of realistic goals for weight loss, healthy eating in general, general nutrition and core food groups, weight management and behavior change strategies for preventing relapse).

Psychological counseling is provided once a week both individually and in group setting. Individual sessions, lasting 45 minutes each, are mainly based on the cognitivebehavioral approach described by Cooper and Fairburn [29] and emphasize the techniques of self-monitoring, goal setting, time management, prompting and cueing, problem solving, cognitive restructuring, stress management and relapse prevention. Group sessions (small groups of 5/6 persons), lasting 1 hour each, focus on issues such as motivation to change, assertiveness, self-esteem, self-efficacy and coping.

Physical activity takes place once a day except for weekend and consists of group programs (20 individuals) based on postural gymnastics, aerobic activity and walks in the open. Patients with specific orthopedic complications carry out individual activities planned by physiotherapists and articulated in programs of physical therapy, assisted passive and active mobilization and isokinetic exercise.

Low to moderate weight losses are expected at the end of the inpatient phase, but notably weight loss is not the primary goal of the inpatient program and each patient is made clear about this point at the very beginning of the treatment. Developing a sense of autonomy and competence are the primary purposes of the inpatient interventions. Patients are afforded the skills and tools for change and are supported in assigning positive values to healthy behaviors and also in aligning them with personal values and lifestyle patterns.

In the last week of hospitalization, just before discharge from the hospital, participants allocated to the TECNOB program are instructed for the out-patient phase. Firstly, they receive a multisensory armband (SenseWear@ Pro2 Armband), an electronic tool that enables automated monitoring of total energy expenditure (calories burned), active energy expenditure, physical activity duration and levels (METs). Patients are instructed to wear this device on the back of the upper arm and to record data for 36 hours every two weeks in a free-living context. The Armband holds up to 12 days of continuous data which the outpatients are instructed to download into their personal computer and to transmit online to a web-site specifically designed for data storing. Outpatients are also told that they can review their progress using the InnerView ${ }^{\circledR}$ Software which analyzes and organizes data into graphs and reports. Secondly, participants are instructed to use the TECNOB web-platform, an interactive web-site developed by TELBIOS S.P.A. (http://www.telbios.it). The TECNOB web-platform supports several functions and delivers many utilities, such as questionnaires, an animated food record diary, an agenda and a videoconference virtual room. In the "questionnaires" section, patients submit data concerning weight and glycated hemoglobin. In the "food record diary" participants submit actual food intake day by day through the selection of food images from a comprehensive visual database provided by METEDA S.P.A. (http://www.meteda.it). The same procedure is also possible through a software called METADIETA (Meteda s.p.a.) previously installed on the outpatients' mobile phones before discharge. Through the mobile phones outpatients maintain the contact with the dietitian who regularly sends them SMS containing syntax codes that METADIETA, the software previously installed into the outpatients' mobile phones, uses in order to visually display the food choices (frequency and portions) outpatients have to adhere according to dietary prescriptions. By this way, outpatients can keep a food record diary allowing comparisons between current eating and the recommended hypocaloric diet along the whole duration of the program. The "agenda" allows the patients to remember the videoconference appointments with the clinicians and the days when to fill in the questionnaires. Moreover, the patients can use the "memo" space to note down any important event occurred to him/her in the previous week/month. Indeed, some research indicates that changes in behavior (eating and exercise) often follow discrete moments [30]. Life events can lead to weight loss but also to weight gain and qualitative research shows that it is not the event per se that results in behavior change but the ways in which this event is appraised and interpreted by the individual [31]. The clinical psychologist has thus the opportunity to discuss with the outpatients about the significant events reported in the "memo" space during the videoconference sessions and cognitively reconstruct dysfunctional appraisals in functional ways. Finally, outpatients are instructed to use the videoconference tool. Thanks to this medium, they receive nutritional and cognitive-behavioral tele-counseling with the dietitian and the clinical psychologist who attended the patients inside the hospital. In particular, just after discharge, participants have 6 videoconference contacts with both clinicians along 3 months. From the 3 rd to the 6th month sessions are scheduled every 30 days and then even more spaced up to an interval of 60 days. During tele-sessions, clinicians (psychologist and dietitian) test the outpatients' progress, their mood, the maintenance of the "good alimentary and physical activity habits", the loss/increase of weight and ask about critical moments, especially those ones reported on the "memo" web-space. In particular, tele-sessions with the clinical psychologist aim to consolidate strategies and abilities acquired during the in-patient phase, to improve self-esteem and selfefficacy, to support motivation, to prevent relapse and to provide problem-solving and crisis counseling. On the other hand, dietitian assesses adherence and compliance to dietary therapy with a special focus on normal eating behavior, sufficient fluid intake, hunger and fullness regulation, appropriate eating/etiquette (pace and timing of meals), slow rate of 
eating, and addresses critical points such as plateau in weight loss or lack of readiness to improve dietary habits.

In addition to videoconference, outpatients can further contact clinicians by e-mail. Indeed, each patient is given the possibility to join his clinician beyond the established videoconference contacts in case of urgency or emergency. According to the e-message's content, clinicians choose the most appropriate format for delivering feedback among email or telephone. In order to avoid excessive dependence and to contain costs, a maximum number of 1 not scheduled contact a week is established a priori.

Great relevance is given to the clinicians-patient relationship as an important medium and vehicle of change [32]. After discharge, out-patients begin to experience the autonomy and competence to change they develop during the inpatient phase and inevitably face resistances and barriers. Thanks to videoconferences, outpatients are supported by the clinicians who attended them during the inpatient phase in exploring resistances and barriers they experience and in finding functional pathways to cope. Furthermore, outpatients are helped to experience mastery in terms of the health behavior change that needs to be engaged.

\section{Measurements}

Primary outcome measure of the randomized trial is weight change in kilograms. Secondary outcome measures are energy expenditure, glycated hemoglobin, binge eating, self-efficacy in eating and weight control, body satisfaction, healthy habits formation, disordered eating-related behaviors and cognitions, psychopathological symptoms and weightrelated quality of life. However, in this paper only weight and disordered eating-related behaviors and cognitions (EDI2) data were analyzed and reported. Weight were assessed with the participant in lightweight clothing with shoes removed on a balance beam scale. The EDI-2 is a widely used, standardized, self-report measure of psychological symptoms commonly associated with anorexia nervosa, bulimia nervosa and other eating disorders. The EDI-2 does not yield a specific diagnosis of eating disorder. It is aimed at the measurement of psychological traits or symptom clusters presumed to have relevance to understanding and treatment of eating disorders. The EDI-2 consists of 11 subscales derived from 91 items. Three of the subscales were designed to as- sess attitudes and behaviors concerning eating, weight and shape (Drive for Thinness, Bulimia, Body Dissatisfaction) and the remaining eight ones tapped more general constructs or psychological traits clinically relevant to eating disorders (Ineffectiveness, Perfection, Interpersonal Distrust, Interoceptive Awareness, Maturity Fears, Asceticism, Impulse Regulation and Social Insecurity) [33, 34].

Follow-up EDI-2 data were self-reported by mailed questionnaires.

\section{Statistical Analysis}

Between-group differences in weight and glycated hemoglobin were examined at all time-points using the MannWhitney nonparametric test due to small sample and violation of parametric assumptions. A completers analysis was performed for any time-points $(n=34$ at 3 months, $n=21$ at 6 months and $n=13$ at 12 months). Changes over time were examined using the Wilcoxon test. Chi-square test was used for categorical variables.

All analyses were performed using the Statistical Package for Windows version 11 for the Social Sciences (SPSS, Chicago).

\section{RESULTS}

\section{Baseline Characteristics}

At baseline, there were no significant differences between groups in age, weight and percentage of females. However, statistically significant differences were found in some EDI-2 scales: Drive for Thinness $(p=0,038)$, Ineffectiveness $(p=0,049)$, Interoceptive awareness $(p=0,003)$, Impulse regulation $(p=0,045)$ and Social Insecurity $(p=0,001)$ (see Table 1).

\section{Weight Change}

There were no significant differences between groups in weight at discharge and at 3-month follow-up. No significant difference was found even in weigh change and percentage of weight loss from baseline to discharge and to 3-month follow-up (Table 2 and Table 3). At 6 and 12 months, completers analysis of weight data did not show any significant difference between groups (Table 2 and Table 3). Moreover, no significant association between groups and number of

Table 1. Baseline Characteristics by Treatment Group

\begin{tabular}{|l|c|c|}
\hline \multicolumn{1}{|c|}{ Characteristics } & TECNOB (n=17) & Control (n= 17) \\
\hline \hline Age, $y$ & $49(46-57,5)$ & $54(49-60)$ \\
\hline Female & $31,3 \%$ & $110(94,9-127,6)$ \\
\hline Weight, Kg & $124(105,6-140,8)$ & $8(3-12,5)$ \\
\hline EDI-2 Drive for Thinness & $4(1,5-7)$ & $5(2-7,5)$ \\
\hline EDI-2 Ineffectiveness & $2(1-4,5)$ & $3(2-10)$ \\
\hline EDI-2 Interoceptive awareness & $1(0-2)$ & 0,038 \\
\hline EDI-2 Impulse regulation & $0(0-2)$ & 0,049 \\
\hline EDI-2 Social Insicurity & $3(1-4)$ & 0,003 \\
\hline
\end{tabular}

Data are given as medians (quartiles) 
Table 2. Medians, Quartiles and Statistical Significance for Between-Group Differences in weight (kg.) at all Time-Points

\begin{tabular}{|c|c|c|c|c|}
\hline & Group & N & Median & Quartiles \\
\hline \hline \multirow{3}{*}{ Baseline Weight } & Control & 17 & 110 & $95-127,6$ \\
\cline { 2 - 5 } & TECNOB & 17 & 124 & $105,6-140,8$ \\
\hline \multirow{3}{*}{ Weight at discharge } & Control & 17 & 105 & $89-121,1$ \\
\cline { 2 - 5 } & TECNOB & 17 & 116 & $101,1-133,5$ \\
\hline \multirow{3}{*}{ Weight at 3 months } & Control & 17 & 105 & $87,4-122$ \\
\cline { 2 - 5 } & TECNOB & 17 & 116 & $99,6-129,3$ \\
\hline \multirow{3}{*}{ Weight at 6 months } & Control & 12 & 104,4 & $91,4-108$ \\
\cline { 2 - 5 } & TECNOB & 9 & 113 & $94,2-125,5$ \\
\hline \multirow{2}{*}{ Weight at 12 months } & Control & 9 & 103,3 & $88,5-114,5$ \\
\cline { 2 - 5 } & TECNOB & 3 & 131 & $88-148$ \\
\hline
\end{tabular}

* Mann-Whitney nonparametric test.

Table 3. Medians, Quartiles and Statistical Significance for Between-Group Differences in Percentage of Weight Loss (kg.) from Baseline at all Time-Points

\begin{tabular}{|l|c|c|c|c|}
\hline & Group & $\mathbf{N}$ & Median & Quartiles \\
\hline \hline \multirow{2}{*}{ Percentage of weight loss at discharge } & Control & 17 & $-4,9$ & $-7,1$ to $-3,7$ \\
\cline { 2 - 5 } & TECNOB & 17 & $-5,2$ & $-6,6$ to $-3,7$ \\
\hline \multirow{3}{*}{ Percentage of weight loss at 3 months } & Control & 17 & $-7,9$ & $-10,8$ to $-2,6$ \\
\cline { 2 - 5 } & TECNOB & 17 & $-6,9$ & $-9,6$ to $-5,4$ \\
\hline \multirow{3}{*}{ Percentage of weight loss at 6 months } & Control & 12 & $-7,3$ & $-12,1$ to 0 \\
\cline { 2 - 5 } & TECNOB & 9 & $-6,9$ & $-10,7$ to $-3,2$ \\
\hline \multirow{2}{*}{ Percentage of weight loss at 12 months } & Control & 9 & $-4,1$ & $-15,3$ to 3 \\
\cline { 2 - 5 } & TECNOB & 3 & $-6,2$ & $-10,6$ to 16,7 \\
\hline
\end{tabular}

* Mann-Whitney nonparametric test.

patients who lost $5 \%$ or more of initial body weight was found at any time-point.

Given the lack of any significant between-group difference, patients were pooled in one group and weight change over time was examined by Wilcoxon nonparametric test. Within-group analyses revealed significant reductions of initial weight at discharge $(-5,9 \mathrm{~kg}, \mathrm{p}<0,000)$, at 3 months $(-8,2 \mathrm{~kg}, \mathrm{p}<0,000)$ and at 6 months $(-7,6 \mathrm{~kg}, \mathrm{p}=0,002)$. Reduction of baseline weight at 12 months was not statistically significant $(-6,9 \mathrm{~kg}, \mathrm{p}=0,136)$.

\section{Eating-Related Behaviors and Cognitions (EDI-2)}

Apart from the variables whose baseline median scores were shown to be statistically different between groups, a significant difference was found in Interpersonal distrust at 12 months $(p=0,02)$. Patients in the control group had a higher median score compared with the TECNOB group (7 vs. 2).

\section{DISCUSSION}

This interim analysis of the first data from the TECNOB study did not reveal any significant difference between the
TECNOB program and a control condition in weight change at 3, 6 and 12 months. Within-group analysis showed significant reductions of initial weight at all time-points but not at 12-month follow-up. The median percentage of initial weight loss for the whole sample was $-5,1 \mathrm{~kg}(-6,6$ to $-3,7)$ at discharge from the hospital. Given the health benefits associated with such weight reductions $[35,36]$, the inpatient program was effective in decreasing weight but notably weight loss was not the primary goal of the in-patient treatment. Beyond medical care, structured diet and physical exercise, obese inpatients received education, professional counseling and skills training from physicians, dieticians, psychologists and physical trainers along the 1-month hospitalization. Empowerment, life-style modification and motivation improvement were the main purposes. Indeed, the great challenge all weight-loss programs have to face is weight-loss maintenance. In this ad interim study, the median percentage of weight loss from baseline was $-7,4 \mathrm{~kg}$ $(-10,1$ to $-5,2)$ at 3 -month follow-up. On average, participants maintained the weight they achieved at the end of the inpatient program and reached further reductions. However, 
completers analysis of data collected at 6 and 12 months showed that participants regained back part of the weight loss and the difference between weight at baseline and at 12month follow-up was no more statistically significant. Notably, sample sizes at 6 and 12 months are small $(n=21$ and $\mathrm{n}=12$ respectively) due to the ongoing status of the study and these results may be unreliable.

Differences in eating-related behaviors and cognitions (EDI-2) were also examined. At baseline, the control group showed higher scores in many EDI-2 scales, i.e. Drive for Thinness, Ineffectiveness, Interoceptive awareness, Impulse regulation and Social Insecurity, compared with the TECNOB group. Notably, these groups included selected participants (those patients that have come through at least the 3month follow-up) and such statistically significant differences were not found when the original groups were compared. Control group showed higher scores also in Interpersonal distrust at 12 months. However, this result has to be seen with caution because of the few patients $(n=12)$ who have achieved the end of the program at present.

In conclusion, these ad interim findings seem not to support the effectiveness of the TECNOB program over a control condition. Notably, this kind of data analysis (ad interim analysis) is underpowered and results we obtained may be unreliable, in particular at 6 and 12 months. However, we gained a significant insight into an important component of the study design, i.e. the 1-month inpatient program. The effect that such uncontrolled factor had on weight loss was very high and probably overwhelmed the effect of the TECNOB intervention. Much statistical power is thus necessary to enhance the chance to detect the effect of the TECNOB program. According to our a-priori sample size calculation [27], 64 participants per group are needed to detect a difference of $3 \mathrm{~kg}$ between the groups at 12 months. Also time is probably a key factor for the detection of the TECNOB effect. Seemingly, the inpatient program has a very high effect in the first months after discharge but such effect may reduce in the long term. A 12-month follow-up is probably sufficient to detect the TECNOB effect over and above the weakened effect of the inpatient program. We will know if our predictions are correct in at least one year.

\section{COMPETING INTEREST}

The technological devices described in this article, except for the web-platform, are commercially available and the authors declare not to hold on any financial interest therein.

\section{ACKNOWLEDGEMENTS}

The TECNOB Project (Technology for Obesity Project) was supported by the "Compagnia di San Paolo" private foundation. Our technological partners are TELBIOS (http:// www.telbios.it) and METEDA (http://www.meteda.it).

\section{REFERENCES}

[1] WHO. Definition and Diagnosis of Diabetes Mellitus and Intermediate Hyperglycemia. Report of a WHO/IDF consultation 2006.

[2] Whitlock G, Lewington S, Sherliker P, et al. Body-mass index and cause-specific mortality in 900000 adults: collaborative analyses of 57 prospective studies. Lancet 2009; 373: 1083.

[3] Flegal KM, Graubard BI, Williamson DF, Gail MH. Excess deaths associated with underweight, overweight, and obesity. JAMA 2005; 293: 1861.
[4] Klein S, Sheard NF, Pi-Sunyer X, et al. Weight management through lifestyle modification for the prevention and management of type 2 diabetes: rationale and strategies. A statement of the American Diabetes Association, the North American Association for the Study of Obesity, and the American Society for Clinical Nutrition. Am J Clin Nutr 2004; 80: 257.

[5] Klein S, Sheard NF, Pi-Sunyer X, et al. Weight management through lifestyle modification for the prevention and management of type 2 diabetes: rationale and strategies: a statement of the American Diabetes Association, the North American Association for the Study of Obesity, and the American Society for Clinical Nutrition. Diabetes Care 2004; 27: 2067.

[6] Colditz GA, Willett WC, Stampfer MJ, et al. Weight as a risk factor for clinical diabetes in women. Am J Epidemiol 1990; 132: 501.

[7] Must A, Spadano J, Coakley EH, Field AE, Colditz G, Dietz WH. The disease burden associated with overweight and obesity. JAMA 1999; 282: 1523 .

[8] Field AE, Coakley EH, Must A, et al. Impact of overweight on the risk of developing common chronic diseases during a 10 -year period. Arch Intern Med 2001; 161: 1581.

[9] Mokdad AH, Ford ES, Bowman BA, et al. Prevalence of obesity, diabetes, and obesity-related health risk factors, 2001. JAMA 2003; 289: 76.

[10] Weinstein PK. A review of weight loss programs delivered via the Internet. J Cardiovasc Nurs 2006; 21: 251

[11] Hill JO, Thompson H, Wyatt H. Weight maintenance: what's missing? J Am Diet Assoc 2005; 105: S63.

[12] Wing RR, Tate DF, Gorin AA, Raynor HA, Fava JL. A selfregulation program for maintenance of weight loss. N Engl J Med 2006; 355: 1563 .

[13] Jeffery RW, Drewnowski A, Epstein LH, et al. Long-term maintenance of weight loss: current status. Health Psychol 2000; 19: 5.

[14] Katan MB. Weight-loss diets for the prevention and treatment of obesity. N Engl J Med 2009; 360: 923.

[15] Donnan PT, MacDonald TM, Morris AD. Adherence to prescribed oral hypoglycaemic medication in a population of patients with Type 2 diabetes: a retrospective cohort study. Diabet Med 2002; 19: 279.

[16] Dale J, Caramlau I, Docherty A, Sturt J, Hearnshaw H. Telecare motivational interviewing for diabetes patient education and support: a randomised controlled trial based in primary care comparing nurse and peer supporter delivery. Trials 2007; 8: 18.

[17] Pagliari C, Sloan D, Gregor P, et al. What is eHealth (4): a scoping exercise to map the field. J Med Internet Res 2005;7:e9.

[18] Eysenbach G. What is e-health? J Med Internet Res 2001; 3: E20.

[19] Castelnuovo G, Gaggioli A, Mantovani F, Riva G. From psychotherapy to e-therapy: the integration of traditional techniques and new communication tools in clinical settings. Cyberpsychol Behav 2003; 6: 375 .

[20] Cline AD, Wong M. New frontiers in using telemedicine for nutrition intervention. J Am Diet Assoc 1999; 99: 1442.

[21] Goulis DG, Giaglis GD, Boren SA, et al. Effectiveness of homecentered care through telemedicine applications for overweight and obese patients: a randomized controlled trial. Int J Obes Relat Metab Disord 2004; 28: 1391.

[22] Jeffery RW, Sherwood NE, Brelje K, et al. P Mail and phone interventions for weight loss in a managed-care setting: Weigh-To-Be one-year outcomes. Int J Obes Relat Metab Disord 2003; 27: 1584.

[23] Maglaveras N, Gogou G, Chouvarda I, et al. Communication infrastructure in a contact center for home care monitoring of chronic disease patients. Proc AMIA Symp 2002: 479.

[24] Rice CA. Prevention: the most economical way to manage diabetes. Nurs Econ 2005; 23: 327.

[25] Saperstein SL, Atkinson NL, Gold RS. The impact of Internet use for weight loss. Obes Rev 2007; 8: 459.

[26] Neve M, Morgan PJ, Jones PR, Collins CE. Effectiveness of webbased interventions in achieving weight loss and weight loss maintenance in overweight and obese adults: a systematic review with meta-analysis. Obes Rev 2010; 11(4): 306-21.

[27] Castelnuovo G, Manzoni GM, Cuzziol P, et al. TECNOB: study design of a randomized controlled trial of a multidisciplinary telecare intervention for obese patients with type-2 diabetes. BMC Public Health 2010; 10: 204

[28] Castelnuovo G. Move the healthcare where it really needs: TECNOB project to improve outpatient obesity treatment. E-letter in response to Wister, Loewen, Kennedy-Symonds, McGowan, McCoy, 
Singer, "One-year follow-up of a therapeutic lifestyle intervention targeting cardiovascular disease risk", CMAJ 2007, 2008; 177: 859-865

[29] Cooper HM. Meta-analysis and the integrative research review. In: Hendrik C, Clark MS, editors. Research methods in personality and social psychology. Newbury Park: Sage 1990; p.142.

[30] Ogden J, Hills L. Understanding sustained behavior change: the role of life crises and the process of reinvention. Health (London) 2008; $12: 419$.

[31] Ogden J, Stavrinaki M, Stubbs J. Understanding the role of life events in weight loss and weight gain. Psychol Health Med 2009; 14: 239 .
[32] Ryan RM, Patrick H, Deci EL, Williams GC. Facilitating health behaviour change and its maintenance: Interventions based on SelfDetermination Theory. Eur Health Psychol 2008; 10: 2.

[33] Conti L. Repertorio delle scale di valutazione in psichiatria. Firenze, Italy: S.E.E., 2002.

[34] Garner DM. EDI-2. Eating Disorder Inventory-2. Professional Manual. Odessa FL, 1991.

[35] Pi-Sunyer FX. Clinical Guidelines on the identification, evaluation, and treatment of overweight and obesity in adults--the evidence report. National Institutes of Health. Obes Res 1998; 6: 51S.

[36] Pi-Sunyer X, Blackburn G, Brancati FL, et al. Reduction in weight and cardiovascular disease risk factors in individuals with type 2 diabetes: one-year results of the look AHEAD trial. Diabetes Care 2007; 30: 1374.

Received: April 13, 2010

Revised: July 02, 2010

Accepted: July 02, 2010

(c) Castelnuovo et al.; Licensee Bentham Open.

This is an open access article licensed under the terms of the Creative Commons Attribution Non-Commercial License (http://creativecommons.org/licenses/by-nc/3.0/) which permits unrestricted, non-commercial use, distribution and reproduction in any medium, provided the work is properly cited. 